\title{
Preserving Health and Achieving Longevity Through Caloric Restriction and Symbiotic Equilibrium with Gut Microbiome
}

\author{
Vinod Nikhra* \\ Department of Medicine, Hindu Rao Hospital and NDMC Medical College, India
}

Submission: January 06, 2019; Published: April 09, 2019

*Corresponding author: Vinod Nikhra, Department of Medicine, Hindu Rao Hospital and NDMC Medical College, New Delhi, India

\begin{abstract}
The Health Preservation and Longevity

The prolongation of life span achieved in recent decades is triumph of modern medicine. The lead challenge now is to promote healthy lifespan along with reducing incidence and severity of chronic and degenerative diseases, and the morbid frailty associated with later years. The host-microbiota interactions can be viewed in the broader context of genetic and epigenetic concepts. The lifestyle changes including dietary alterations accompanying aging have impact on gut microbiota and the activity of the innate immune system, intern, is influenced by gut microbiota. The gut microbiota also modulates cardiometabolic and inflammatory processes, thus influencing aging process.
\end{abstract}

Caloric Restriction and Gut Microbiome

There is evidence that optimal CR along with adequate nutrition can reduce adiposity, chronic inflammation and insulin resistance, and promote health and quality of life along with satisfactory level of activity of daily living (ADL) in older adults. Further, as documented by recent research, the loss of gut microbiota diversity occurs during later years and modulates aging process adversely. The loss of core microbiota diversity with advancing age, has been associated with increased frailty and degenerative diseases along with cognitive decline.

\section{Gut Microbiota and Immunosenescence}

Diet-microbiota-health interactions and the lifestyle changes including dietary alterations accompanying aging have impact on gut microbiota. Since the gut microbiota modulates cardiometabolic and immunological processes, the microbiota alterations with aging mean that older adults may experience accelerated aging-related health loss. Further, the age-associated alterations in composition, diversity and functional aspects of gut microbiota have been related to age-associated decline in immune system functioning (immunosenescence) and low-grade chronic inflammation (inflam-aging), which accompany various aging-associated pathologies.

\section{Conclusion-Scope of Interventions}

The gut microbiota has crucial impact on aging process, and as documented by recent studies the microbiota-targeted interventions for normalization of gut microbiota may have health-span-promoting effects. The CR with adequate nutrition and microbiota-targeted dietary and probiotic, prebiotic and symbiotic interventions are likely to favourably modulate the host health and aging process by enhancement of antioxidant activity, suppression of chronic inflammation and improvement of immune homeostasis and cardiometabolic profile.

Keywords: Aging process; Caloric restriction; Chronic degenerative diseases; Cognitive decline; Gut microbiome; Immunosenescence; Inflamaging; Longevity; Microbiota-targeted interventions; Prebiotic; Probiotic

\section{Microbiome, General Health And Longevity}

\section{The Human Gut Microbiome}

The human gut is inhabited by over 100 trillion microbes of about 1000 species. These microbes have co-evolved with humans over millennia and live together in symbiotic state. The most populous bacterial phyla are Bacteroidetes and Firmicutes, constituting more than $90 \%$ of the gut microbiota, and various species in lower abundance constitute the remainder. The gut microbiota is a complex community of bacteria residing in the GIT, the bacterial load is low in the stomach and increases expo nentially from the duodenum, the jejunum, and the ileum, to the colon, harbouring about 109-1013 bacteria [1]

The gut microbiota encodes over 150 times more genes than the human genome and influences the host physiology and homeostasis, in addition providing numerous metabolites for maintenance of intestinal and general health. The activity of the innate immune system is also influenced by various subgroups of gut microbiota. This dynamic population of millions of microbes is called microbiome, having almost 10 bacterial cells for every one of human cells, which together form the meta-organism. 
The lifestyle changes including dietary alterations accompanying aging have impact on gut microbiota. The alterations in gut microbiota, which modulates cardiometabolic and inflammatory processes, the alterations with aging mean loss of various physiological functions leading to accelerated aging-related health loss in older adults. There occur changes in core microbiota taxa leading to low microbial diversity and gut dysbiosis. Though, the core microbiota may decline and be supplemented by other abundant species, the full core microbiota is rarely lost (Figure 1).

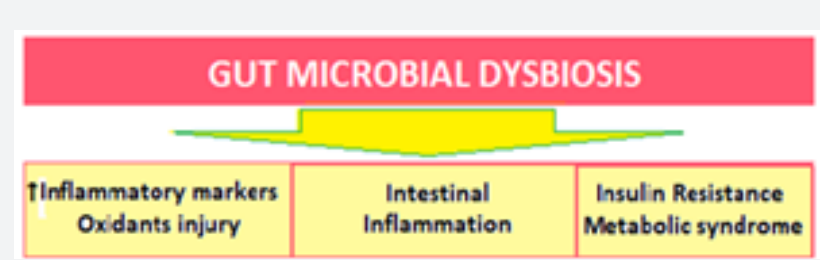

Figure 1: The Relationship between Gut Dysbiosis, Chronic Inflammation and Metabolic syndrome

These changes lead to increase in inflammatory markers and oxidant injury, lead to intestinal inflammation and insulin distance (IR), precipitating T2DM and metabolic syndrome. The group of organisms that are mostly affected by aging, are the diversity-associated taxa, comprising of Privately and associated genera [2]. Further, the extreme-aged older adults (centenarians) have a microbiota different from that in older adults [3]. However, several specific co-abundant taxa seem to be associated with old age and malnutrition.

\section{The potential effect on health}

The potential for the gut microbiota to affect health has a particular relevance for older adults. The age-associated loss of diversity in the core microbiota groups is associated with changes in innate immunity, sarcopenia and cognitive function, bringing-about increased frailty and reduced cognitive performance with aging. There is no threshold of age at which the composition of the microbiota alters; rather, the changes occur gradually. Thus, the gut microbiota of older adults differs from that of younger adults. Further, the degree of retention of the core microbiome is associated with age, general health and care, and dietary factors [4].

As established by several recent studies, the gut microbiota has a crucial impact on the aging process, and, the microbiota-targeted interventions for normalization of gut microbiota may have health and lifespan-promoting effects. The host-microbiota interactions can be viewed in a broader context of genetic and epigenetic concepts. It depends on the host nutrient signalling pathways for its effects on host health and lifespan. The inter-relationship of microbiome and human body is complex, the genomes within the meta-organism interact and affect one another and have impact on human physiology and homeostasis. The influence of gut microflora on host health comprises of two extremes of the relationship: symbiosis and pathogenesis.
The most of gut microbes are commensal, i.e., neutral, and genomic sequencing have led to characterization of the diverse commensal bacteria, comprising the metagenome - the ensemble of host and microbiota DNA, and by extension the meta-transcriptome, proteome and metabolome. Further, the microbiota extends its effects beyond host pathophysiology, to modulation of the aging process [5]. The gut microbiota shows a wide inter-individual variation, but intra-individually, it is relatively stable over time [6]. There is a functional core microbiome, provided by the abundant bacterial taxa which is common to human hosts regardless of age, gender and geographic location.

There is an inter-individual variation in the gut microbiota profile, but major constituent microbes are common. The core microbiome consists of regularly present taxa - the enterotypes made up of 3 distinct microbial metagenomic clusters being Bacteroide, Prevotellaceae and Firmicutes (Ruminococcaceae and Lachnospiraceae). With age, the gut microbiota becomes diverse and variable, simultaneously certain groups of bacteria associated with frailty increase. The gut microbiome communicates with the host through various biomolecules, nutrient signaling-independent pathways, and epigenetic mechanisms. Disturbance of these communications associated with age-related gut dysbiosis can affect the host health and lifespan. The gut dysbiosis disrupts the symbiosis and interdependence and triggers the innate immune response and chronic low-grade inflammation, leading to suboptimal aging, age-related frailty and chronic degenerative diseases.

\section{The age-related alterations}
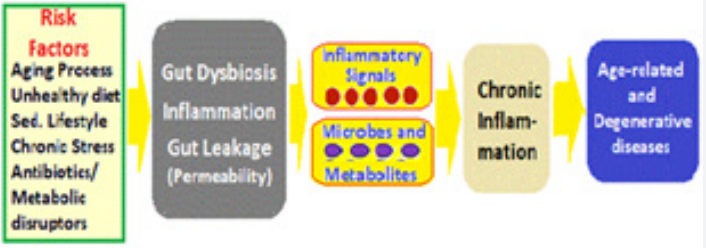

Figure 2: The Relationship between the Risk Factors, Gut Dysbiosis and Chronic Inflammation, and Age-related and Degenerative diseases.

With the age the composition of microbiota changes due to senescence of the gut and risk factors including the altered physiological environment with age, the lifestyle factors and diet playing a major role (Figure 2). With aging, there is often reduction in amount of food and associated malnutrition [7]. There is decrease in variety of fibre-containing foods consumption leading to a decrease in microbiota diversity particularly with the abundant Clostridial subpopulation [8]. The core microbiota decreases in abundance with aging, its loss playing a crucial role in the aging process [9]. The studies in fruit fly, Drosophila melanogaster, have documented the correlation between composition of food intake and diversity of the microbiota [10].

The microbiota composition alters, becoming less diverse and more instable over time in the older adults [11]. Further, 
microbiota alterations and greater than normal loss of gut microbiota diversity, occurring with aging entail that older adults experience accelerated aging and age-related health loss and frailty. The loss of Clostridial subpopulation is also significantly associated with increased frailty. After an initial change, microbial diversity tends to recover among people living in the community and less well in those in long-stay residential care [12].

The extreme-aged older adults (centenarians), in general, have an overall decline in microbiome function parallel with various alterations in the loss of physiological functions [13]. With longevity, in centenarians, there occurs enrichment of subdominant taxa. The longevity adaptation seems to involve enrichment in health-associated gut bacteria [14]. Centenarians are a model for healthy aging because they have reached the extreme limit of life by escaping, surviving, or delaying chronic diseases. The gut microbiota in centenarians differs from that in older adults [15].

\section{The Host-Microbiota Symbiotic Co-Existence}

\section{The loss of microbiota with age}

Once established early in life around 3 years of age, gradually after birth, the human gut microbiota is stably maintained depending on the host's dietary and health conditions. Its communication with the host systems, microbiota-host crosstalk's, involves various signaling networks and their mediators. In addition, the gut-brain axis connects gut microbiome with the central nervous system via neurons, hormones or cytokines.

The cumulative abundance of core microbiota consisting of symbiotic bacterial taxa (mostly belonging to Ruminococcaceae, Lachnospiraceae, and Bacteroidaceaefamilies families), decreases with age, and there occurs an increasing abundance of subdominant species, as well as a rearrangement. These features are maintained during longevity and extreme longevity, when certain opportunistic and allochthonous bacteria such as Akarnania, Bifidobacterium and Christensenellaceae turn into commensal and support health. These bacterial taxa then become involved in establishing of a new homeostasis with the aging host and contribute in aging-associated alterations in composition, diversity and functional attributes of gut microbiota [16]. The age-related decline in immune system functioning (immunosenescence) and a low-grade chronic inflammation (inflam-aging), may accompany aging. Because of its impact on human physiology, metabolism and immunity, the gut microbiome is a potential determinant of healthy aging [17]. Indeed, the preservation of host-microbes homeostasis can counteract inflam-aging, intestinal permeability, and decline in muscle and bone mass and cognitive health [18].

\section{Studies in invertebrate models}

The simpler organisms, fruit fly (D. melanogaster) and the nematode worm (Caenorhabditis elegans) also live in the presence of microbiota both in wild and lab habitat, like other more complex organisms. Due to their genetic tractability and short lifespans, the studies allow to document the effect of microbiota on genetic pathways modulating the aging process. The host and bacteria interactions, thus, extend beyond intestinal homeostasis to influence systemic health and longevity.

C. elegans are grown in co-culture with non-pathogenic strains of E. coli 'OP50' bacteria, which provides essential nutrients and components to it. The non-pathogenic, E. coli OP50 proliferate inside older animals and suppresses infection and increases worm lifespan [18]. In young worms however, E. coli are mechanically broken down before entering the gut and do not live in the animal as a true microbiome. Beyond their role as a nutrient source, E. coli secrete diffusible molecules, including metabolites and small RNAs that can directly impact C. elegans aging. C. elegans cannot produce their own NO since they lack NO synthase.

The worms have been shown to utilize NO produced from the bacteria within their microenvironment and the bacterial NO modulates C. elegans lifespan via effects on host transcription [19]. Bacterial-derived signals NO and ncRNAs regulate C. elegans longevity. E. coli modulate longevity of the worm by using the innate RNAi machinery, as the worm incapable of RNAi are resistant to the effects of Dra. Metformin increases C. elegans lifespan via effects on bacterial folate metabolism: Feeding worms metformin robustly increases lifespan in an AMPK-dependent manner. This response is indirectly mediated by the effect of the drug on bacteria, rather than the worms themselves.

Metformin suppresses folate metabolism specifically in E. coli, and feeding worms increases lifespan. Reduced folate metabolism decreases methionine content of bacteria, and this effect is causal to metformin-induced longevity in C. elegans. L. plantarum drive Drosophila growth under low nutrient conditions via the longevity modulator TOR. The bacteria also affect the capacity of flies to sense nutrients. The presence of microbiota at the interface of the gut epithelium also has systemic effects on host aging. The PGRP-SC2 overexpression in Drosophila ECs significantly increases lifespan dependent upon the presence of commensal bacteria in the gut. The reduced dietary methionine has been shown to promote longevity in both Drosophila and rodents. Metformin decreases folate levels in humans, as well.

\section{Gut microbiota and aging process}

In general, there occurs compositional change in the functional core microbiome and/or the enrichment of non-core functions with advancing age [20]. In fact, the changes in composition of gut microbiota and gut microbial diversity inversely correlates with biological age or functional age and are independent of chronological age. The gut microbiota of the elderly becomes more diverse and variable with advancing age and the three main bacterial taxa in the core microbiota, become less abundant in older age groups, while certain health-associated species become more abundant in older age groups including centenarians and semi-supercentenarians. In general, the Ruminococcin and Corbeilles (Firmicutes phylum), and Egger Thella (Actinobacteria) genera becomes abundant, the overall gut mi- 
crobiota richness decreases, while some microbial taxa associated with unhealthy aging emerge with increase in biological age.

\section{Gut Dysbiosis And The Aging Process}

The gut dysbiosis is the disruption of the commensal homeostasis between the host and gut microbiota due either altered host factors or microbial composition or both. The factors contributing to gut dysbiosis include unbalanced diet, environmental toxins, drugs, ROS, psychological stressors and various proinflammatory conditions. Gut dysbiosis due to antibiotics or high-fat or carbohydrate intake is associated with obesity and metabolic disorders. In turn, the gut dysbiosis has been associated with disorders like inflammatory bowel disease, obesity, diabetes, cardiovascular diseases and neurodegenerative diseases. The altered innate immune response caused by dysbiosis, in the aging gut provokes chronic inflammation and oxidative injury leading to gut dysplasia and defective epithelial functioning, and accelerated aging and increased mortality.

\section{The concept of inflam-aging}

With advancing chronological age, the homeostatic relationship between the gut microbiota and the host deteriorates along with gut dysbiosis and the gut microbiota becomes more diverse with phylogenetic richness. The dysbiosis changes in the aging gut provoke oxidative stress and proinflammatory changes with altered innate immunity. In addition, the gut dysbiosis disturbs communication between the gut $\neg$ microbiota and the host through various biomolecules, CR-independent signaling pathways and epigenetic mechanisms, promotes proinflammatory immune responses and chronic degenerative reactions affecting the host health and longevity.

There is an evidence from the research that the chronic, age-related inflammation called inflam-aging is brought about by senescent cells, cell debris immunosenescence and microbial burden. Thus, the age-associated changes in intestinal microbiota and their bidirectional relationship with the host, bring about chronic inflammation. The gut microbiome appears to play a key role in age-related inflammation [21]. With the advanced age, the ability to resolve inflammation becomes impaired leading to sustained tissue infiltration by leukocytes and chronic release of pro-inflammatory cytokines and chemokines.

\section{The concept of immuno-sconce}

The most important factor associated with age-related inflammation is decrease in the efficiency of the immune system, i.e., immuno-senescence, characterized by thymus atrophy, reductions in neutrophil function, naïve $\mathrm{T}$ cell number, and the cytotoxic capacity of natural killer cells, and lowered B-cell antibody production in response to antigen. The cause of immune-senescence is thought to be that the chronic lifetime antigenic burden exhausts finite capacity of the organism's immune system, leading to long-term risks of chronic inflammation and disease. The gut microbiome appears to play an integral role in these age-related inflammatory changes. The advanced age is as- sociated with changes in microbiota composition characterized by a loss of diversity in the core taxa associated with key geriatric syndromes including physical frailty and cognitive decline.

\section{Gut dysbiosis and animal models}

The research studies in invertebrate and vertebrate species have led to understanding of various aspects of the pathophysiology of gut dysbiosis. Through the loss of gut microbial richness and altered microbiome composition, dysbiosis has been documented to play a significant role in accelerated aging process and negative impact on longevity.

1. In the African turquoise killifish, loss of the gut microbial richness occurs with aging. By treating the middleaged killifish with antibiotics to prevent the age-related gut dysbiosis and with intestinal contents from the young fish, Smith et al were able to show significant lifespan extension. Further, the long-lived recipient fish maintained higher mobility and microbial diversity compared with controls [22].

2. In Drosophila, there is increased number of gut microbes in older flies. The gut dysbiosis triggers intestine epithelial dysplasia and altered innate immune response in aging flies and leads to increased mortality [23].

3. The mice studies also confirm the causative role of gut dysbiosis in innate-immunity-induced inflammation.

Transfer of the gut microbiota from old mice to young germfree mice triggers altered innate immunity and inflammatory response mimicking "inflam-aging" [24].

\section{Impact of biomolecules from microbiota on aging pro- cess}

The commensal microbes produce numerous biomolecules endogenously by in the digestive tract, which modulate health and aging process. These include various vitamins, fermentation products, gut-derived hormones and bio-compounds relevant to the neurological health.

1. Rapamycin is an immunosuppressant produced by soil bacteria which binds to immunophilin, an FK binding protein, and the complex inhibits mTOR function. In mice, it alters the host gene expression profiles as well as the gut metagenomes. The FOXO, regulating expression of various proteins, is an important factor for aging process and lifespan. The inactivation of FOXO as well as its chronic activation shortens lifespan, the overall effect being the net effect of all the positive and negative effects combined. Thus, chronic activation of IMD/Relish or inactivation of dual oxidase results in shortened lifespan in fruit flies.

2. Metformin is a compound of exogenous origin. In C. elegans, excessive folate limits lifespan and metformin extends lifespan through inhibition of bacterial folate and methionine metabolism.

3. Nitric oxide (NO) is a signaling molecule involved in many physiological functions. C. elegans cannot produce NO due 
to lack of the NO synthase. NO provided either by gut bacteria or by exogenous supplementation increases lifespan.

4. Colonic acid, an exopolysaccharide, promotes mitochondrial fission and enhances the mitochondrial unfolded protein response under stressful conditions. The beneficial effects of colonic acid are independent of the CR signaling pathways. It extends lifespan in C. elegans and D. melanogaster.

5. Short-chain fatty acids (SCFAs) are fermentation products of dietary fibers by the anaerobic gut microbiota, which enter the circulation from the gut and have certain beneficial roles in energy metabolism. Acetate reduces serum cholesterol and triglyceride levels, propionate lowers glucose levels, and butyrate improves insulin sensitivity. There occurs age-related decline in the concerned genes and in SCFAs production, which has been associated with frailty.

SCFAs have both negative and positive effects on health, in a dose dependent manner. Some SCFAs are the main causal factor for the $\alpha$-synuclein-related pathology caused by the gut microbiota. In the mouse model of Parkinson's disease, $\alpha$-synuclein aggregates activate immune cells, including phagocytic microglial cells in the CNS and treatment of germ-free mice overexpressing $\alpha$-synuclein with a mixture of acetate, propionate and butyrate leads to neuroinflammation and motor deficits [25].

Another SCFA, butyrate inhibits histone deacetylases (HDACs) and has a profound effect on the host epigenome and aging process. The inhibition of HDACs by butyrate promotes histone lysine acetylation, leading to an open chromatin state and transcription activation. Butyrate increases lifespan in Drosophila. In aging mice, butyrate counters muscle atrophy and enhances memory functioning [26]. The activation of FOXO by HDACs can cause skeletal muscle atrophy in mice via autophagy, and inactivation of HDAC by butyrate can reverse the atrophy.

\section{Gut dysbiosis and neurodegeneration}

The gut microbiota is also causally linked to the development of neurodegenerative disorders [27]. The enteroendocrine cells in gut epithelium express $\alpha$-synuclein, which is close to $\alpha$-synuclein-containing enteric neurons, prompting the hypothesis that $\alpha$-synuclein originates from the gut and spreads to the central nervous system and may be causative for Parkinson's disease [28]. Cerebral deposition of $A \beta$ plaques is a critical risk factor for various types of dementia, including Alzheimer's disease. The causative role of the gut dysbiosis in Alzheimer's disease has been conceptualised.

Transgenic mice expressing $A \beta$ precursor protein start to accumulate cerebral $A \beta$ early on, and their gut microbiota composition differs greatly compared with that of the non-transgenic littermates. Transgenic mice rendered germ free show lower $A \beta$ levels and significantly reduced cerebral $A \beta$ deposition compared with conventionally reared transgenic mice. Furthermore, transfer of the gut microbiota from conventionally reared transgenic mice to germ-free transgenic mice was more effective in the induction of $A \beta$ pathology. These observations indicate the role of the gut brain axis in development of neurodegenerative disorders [29].

\section{Factors Related to Dysbiosis and the Host Health}

\section{Gut dysbiosis and disturbed homeostasis}

An enormous portion of immune system is involved for maintaining homeostasis with the microbiota and about $70 \%$ of the total lymphocytes reside in the gut-associated lymphoid tissue. Further, the gut microbiota is significantly involved in various inflammatory responses and is an important regulator of immunity including tolerance. The microbiota suppresses inflammatory responses to food and other orally ingested antigens and play a protective role against inflammatory responses through toll-like receptor (TLR) activation to promote tissue repair and survival. The immune cells also regulate inflammation and allergic reactions through recognition of SCFAs produced via bacterial breakdown of indigestible dietary components such as fibre.

Though the human ageing process is associated with gradual declines in functions across almost all organs, the bacterial organisms in the gut do not age per se. However, due to various age-related functional alterations, the older adult manifest changes in lifestyle patterns, diet and other gut-related physiological functions influencing the gut microbiota composition and stability. The high-fat diet can potentially alter gut bacteria and leads to dysbiosis, which contributes to increased gut permeability and metabolic endotoxemia, contributes to low-grade inflammation and insulin resistance and diabetes, adiposity and other metabolic disorders (Figure 3).

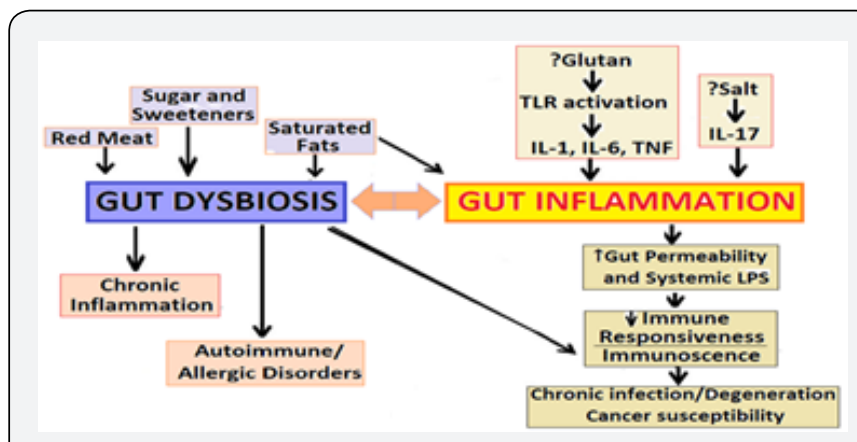

Figure 3: Genesis of Gut Dysbiosis and Chronic Gut Inflammation, and the Autoimmune, Age-related and Degenerative diseases.

\section{Impact of Dysbiosis on Physical Health and Immunity}

Gut dysbiosis and physical frailty: The recent research suggests that the gut microbiome plays a significant role in age-associated physical frailty in older adults [30]. The age-related dietary changes also appear to contribute to the dysbiosis. Advanced age associated with deterioration in nutrient intake and absorption including dentition, sensory changes like taste and smell, salivary function, digestion, and intestinal transit time also contribute to dysbiosis. The host factors like unhealthy diet and use of medications, especially antibiotics, have impact on the gut microbiota composition and function and contrib- 
ute to gut dysbiosis, a state of disrupted homeostasis of the gut microbiome, which leads to increased IR and may manifest as adiposity and T2DM [31,32]. Further, adiposity and T2DM are associated with an altered gut microbiota, inflammation, and gut barrier disruption.

Gut dysbiosis and immunity: The changes in gut microbial diversity and density leading to dysbiosis have been shown to have impact on immunity leading to chronic inflammation, even in the distal organs. The failure to regulate the inflammatory responses is a contributor to the development of various chronic inflammatory and degenerative disorders affecting the gut and other organs including the central nervous system and contributes to age-related cognitive decline. The brain amyloid content and circulating inflammatory analytes have been associated with the inflammatory bacteria taxon Escherichia/Shigella and inversely associated with the anti-inflammatory E. rectal taxon. A recent study has linked brain amyloidosis and chronic inflammation among cognitively impaired elders with the abundance of pro- and anti-inflammatory gut microbiota [33].

There appears to exist a gut microbiota signature that promotes intestinal inflammation and subsequent systemic lowgrade inflammation, which in turn promotes the development of T2DM [34]. The animal studies have also endorsed that shifts in the composition of the gut microbiome influence metabolism and energy balance, and the chronic inflammation and increased gut permeability may play a significant role in development of adiposity and T2DM [35]. The immune system is brought up by commensal bacteria, especially bacteria in the gut. Homeostasis of the gut microbiota is important in modulation of the host immunity and control of inflammation [36].

The fallouts of gut dysbiosis: The gut microbiota-derived metabolites, lipopolysaccharides (LPSs) and SCFAs have impact on IR and adipogenesis $[37,38]$. LPSs cause low-grade inflammation through the induction of inflammatory cytokines by immune cells and adipocytes. Whereas SCFAs, which are end products of the microbial fermentation of macronutrients such as acetate and butyrate, modulate gene expression and reduce chemokine and proinflammatory cytokine production by monocytes, acting on intestinal tissue immune cells locally as well as systemically. The high-calorie diets contribute to obesity and T2DM, and there appears to exist a link between diet, obesity and the gut microbiota.

The studies in mice have demonstrated that a high-fat diet (over $60 \%$ calories derived from fat) decreases the gur microbial diversity. In an important study, Le Chatelier et al documented that the diversity of human gut microbiome correlated with IR, fatty liver and increased C-reactive protein and leptin concentrations and decreased serum adiponectin concentrations in both nonobese and obese Danish individuals [39].

The age-related changes in microbiome, the increased use of medications including antibiotics and consumption of high-sat- urated fat and high-sugar diet appears to contribute to the depletion of certain beneficial components of the microbiome [40]. This, in turn, leads to to chronic activation of the immune system, altered immunity due to chronic activation of innate and adaptive immune systems. The increased intestinal permeability is associated with IBDs and other disorders linked with the gutbrain axis.

Gut dysbiosis - Changes with age: There occur changes in the intestinal epithelial barrier with age [41]. The gut barrier and nutrient transport functions decline with aging, and dysbiosis weakens the intestinal barrier function. In the Irish ELDERMET cohort study, there has been documented alterations in the core microbiota of those over 65 years of age, characterized by a greater proportion of Bacteroides spp. and distinct abundance patterns of Clostridium groups compared to younger individuals [42]. The older individuals also display a loss of diversity-associated taxa, including Privately and associated genera, contributing to instability in the microbiome composition. In another study, Biaggio et al. reported that a group of centenarians from Northern Italy displayed low species diversity compared to younger adults $\sim 30$ years of age. The specific changes occur within Firmicutes (one of the two dominant phyla in the gut) subgroups and enrichment of Proteobacteria - an opportunistic, which can overtake commensal bacteria and induce pathology.

These microbiome changes are also accompanied by loss of genes for SCFAs production and decreased saccharolytic potential, while proteolytic functions become more abundant compared to the intestinal metagenome of younger adults [43]. Further, these changes in microbiota are associated with increased plasma concentrations of inflammatory cytokines IL-6 and IL-8.

These observations are supported by the studies in Drosophila, documenting that age-related changes potentiate chronic inflammation and increased intestinal permeability [44]. The mice studies by Devarajan et al, also support this hypothesis [45]. In the study, the germ-free mice did not display an age-related increase in systemic pro-inflammatory cytokines, but co-housing germ-free with old and conventionally raised mice increased circulating pro-inflammatory cytokines. Further, anti-TNF therapy reversed age-related microbial changes.

\section{Gut Microbiota-Health and Longevity}

\section{Future trends and scope of interventions}

The scope of interventions: As documented by recent studies, the gut microbiota has a crucial impact on the health and aging process. Further, the microbiota-targeted interventions for normalization of gut microbiota and restoration of symbiosis appear to have potential health-span-promoting effects [46]. The successful execution of the targeted interventions for normalization of gut microbiota needs Diagnostic tests, followed by nutritional therapy and microbial modulation, followed by the post-therapy assessment (Figure 4). 


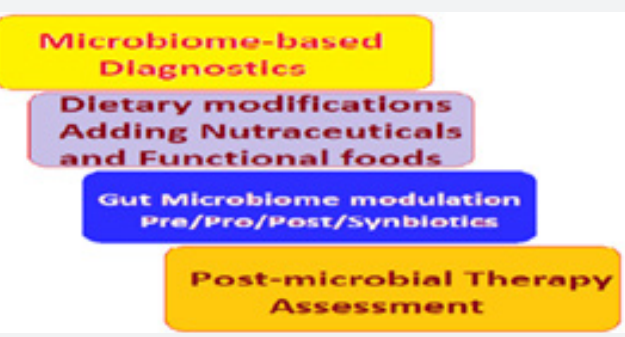

Figure 4: The steps for targeted interventions for normalization of gut microbiota: Diagnostics, Diet modulation and nutritional therapy, Microbial modulation and Post-therapy assessment.

The microbiome-based diagnostic tests should be followed by lifestyle changes including exercise and upgradation of activity level and dietary modifications by planning a balanced diet rich in functional foods and adding nutraceuticals. The measures for the gut microbial modulation by prebiotics, probiotics and symbiotic, should be followed by the post-microbial therapy assessment, and suitable action thereafter. The normalization of gut microbiota and restoration of symbiosis between microbiome and the host is a continuous endeavour for health and longevity.

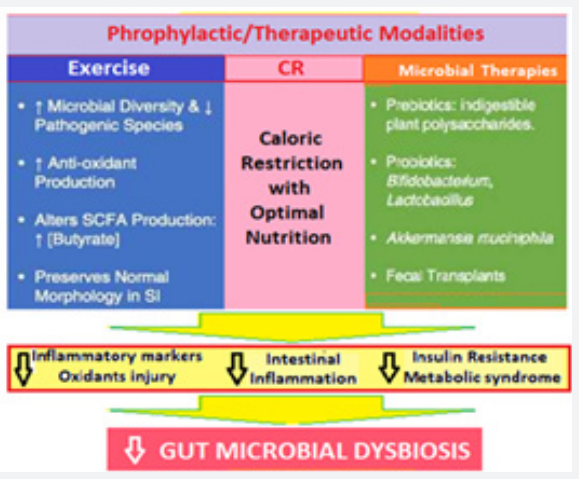

Figure 5: The prophylactic and therapeutic modalities to decrease oxidative injury, intestinal inflammation, dysbiosis and $\mathrm{IR}$, and for normalization gut microbiota.

The microbiota-targeted interventions, thus, appear to be a promising health-preserving, anti-aging treatment plan (Figure 5). Various health-span-promoting interventions include both prophylactic and therapeutic modalities to decrease oxidative injury, to curtail chronic intestinal inflammation, improve gut dysbiosis and insulin resistance and its fallouts, and for normalization gut microbiota to restore symbiotic relationship with the host.

Potential intervention strategies: The microbiota-targeted dietary and probiotic interventions (Figure 6) have been shown to favourably affect the host health and aging by an enhancement of antioxidant activity, improving immune homeostasis, suppression of chronic inflammation, regulation of fat deposition and metabolism and prevention of insulin resistance [47]. Certain simple measures to support the gut microbiota include - the dietary intake of local, seasonal vegetables and fresh fruits, and organic foods, along with a fair portion of healthy fats.

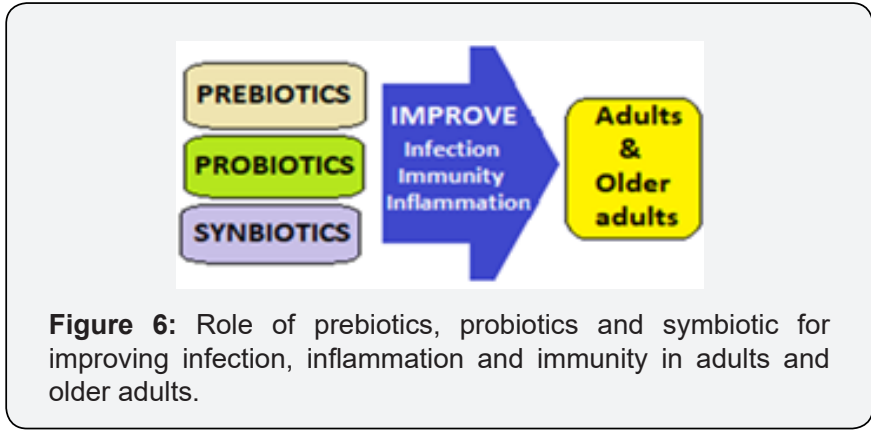

This should be accompanied with along intake of probiotic and prebiotic-rich diet containing plain yogurt, aged cheeses and fermented foods such as kimchi, and avoiding exposure to antibiotics and unnecessary medication including laxatives. Further, there are potential benefits from nutritional supplements like coffee, resveratrol and quercetin and other flavonoids. Similarly, the Mediterranean diet hold promises for balancing the gut microbiome [48].

It appears that interventions designed to target the gut microbiome may be capable of producing beneficial effects on age-related chronic inflammation and gut dysbiosis, and overall health. Some of the promising strategies having beneficial effects on the gut microbiome are:

I. Nutrition and lifestyle factors: The gut barrier and nutrient transport functions decline with aging and microbial dysbiosis adversely affects the intestinal barrier function. In addition, nutrition is the major factor affecting the gut microbiota as well as influencing the host's epigenetic milieu. The nutrition derived factors such as folate and choline, as dietary methyl-donors, influence DNA methylation and the nutrient-signaling pathways.

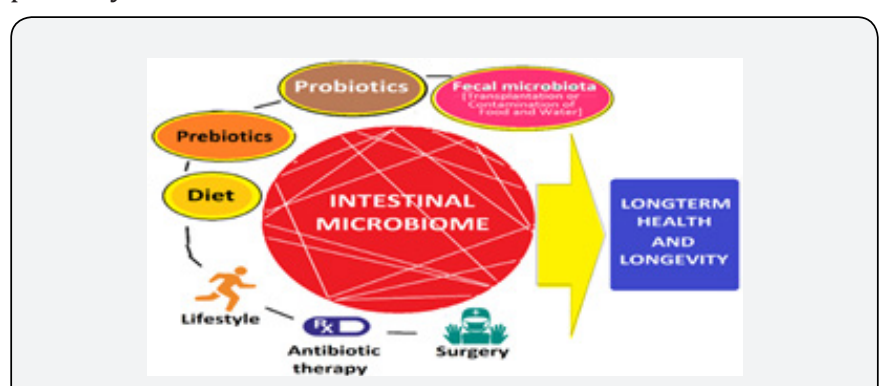

Figure 7: The dietary and lifestyle factors influence gut microbiome; Prebiotics, probiotics and fecal microbiota loading may have positive impact; and Antibiotic therapy and bariatric surgery alter microbiota equilibrium.

The high-fat diet appears to be one of the driving factors for gut dysbiosis, inversely the diet low in fat content is helpful (Figure 7). The moderate reduction in calorie intake, i.e., caloric restriction (CR) potentially improves health and extend lifespan through certain evolutionarily conserved biological pathways.

The reduced nutrient availability, brought about by $\mathrm{CR}$, decreases AKT (protein kinase B) activity resulting in enhanced FOXO (Forehead box 0 transcription factor) activity. Inversely, the activation of AKT by nutrient abundance leads to the inacti- 
vation of FOXO, a major factor for inducing expression of various pathways involved in cell metabolism, autophagy and stress-response, an essential link to longer lifespan [49].

The gut-specific FKH, a homolog of Forehead box A (FOXA) upregulation has been shown to improve gut barrier function and expression of nutrient transporters, which help to increase lifespan. The Physical exercise and increase in activity of daily living (ADL) is clinically recommended intervention having important benefits on the gut physiology and microbiota [50]. The aerobic exercise enhances epithelial membrane integrity, improves microbial diversity and attenuates intestinal inflammation.

II. Probiotic-rich and Fermented foods: Interventional studies show that dietary changes result in substantial and rapid changes in the make-up of the gut microbiome [51]. The deprivation of fermented foods in diet causes a fall in innate immune response, including decreased phagocytosis by leukocytes and immune response against infections [52]. The consumption of probiotic bacteria such as those found in yogurt and other fermented milk products have beneficial effect on composition of the gut microbiome [53].

The dietary supplementation with probiotics is one of the most promising interventions for re-balancing the gut microbiome. Supplementing with probiotics supports the gut microbiome and help in repopulating gut with beneficial bacteria. The well-known actions of probiotics include antimicrobial activity, improved gut microflora composition, enhancement of intestinal barrier function and immunomodulation. A study in mice has reported that probiotic supplementation with Lactobacillus plantarum WCFS1 prevented age-related decline in the colon mucus barrier in a mouse model of accelerated aging [54]. The probiotics have anti-inflammatory properties and can also be utilized as a vehicle to deliver other therapeutic compounds [55].

III. Fecal microbe transplantation: Recently, a high effectiveness and safety of novel therapeutic application such as fecal microbiota transplantation in the prevention and treatment of age-related pathological conditions including atherosclerosis, T2DM and Parkinson's disease has been demonstrated.

IV. Others: Metformin prevents the overproduction of glucose by hepatocytes, it delays glucose absorption during digestion after a meal. The administration of metformin appears to alter the composition of the microbiota. It increases abundance of mucin-degrading bacterium Akarnania in the obese mice. A recent study in human volunteers has confirmed the effect of metformin on the gut microbiota.

Modification of gustatory or olfactory neurons, apart from nutrient availability, the has been shown to modulate lifespan in Caenorhabditis elegans and Drosophila melanogaster. Studies support the notion that gastric bypass surgery leads to a substantial shift in the gut microbiota, which may contribute to weight loss.

\section{Treatment for chronic inflammation}

Treatments for chronic inflammation appears to be a promising strategy for healthy aging. The gut microbiome may represent a novel site of intervention for the prevention and treatment of late-life inflammation. A variety of biological, medical and lifestyle factors appear to contribute to gut dysbiosis in late-life, and interventions specifically designed to target these factors may be useful in restoring microbial balance and attenuating inflammation. The healthy aging is dependent not only on genetics, lifestyle choices and a positive attitude, but also another fast emerging factor, the gut microbiome. The latter plays a significant larger role in staying healthy in older adults. Further, In one of the largest studies on human gut microbiota, has documented that the composition of the gut microbiome of healthy older adults was essentially similar to that of healthy younger people [54-56]. But, the gut microbiomes of the elderly have several characteristics, including instability among gut bacterial species counts and reduction in bacterial diversity [55].

The unbalanced microbiome appears to be related to various harmful alterations in health, whereas the healthy older adults and elderly (up to 90 years of age) have the rich and thriving microbiome similar to younger people who are about 30 years old. The studies reinforce the importance of balanced microbial diversity for health and that some of the unavoidable consequences of aging might be the reversible effects of an unbalanced microbiome. Further, various studies have shown that it may be possible to reset the microbiome to that of a younger and healthier person.

The composition of gut microbiome naturally shifts over time and swings towards having more Bifidobacterial with aging. This natural phenomenon, however, is hampered by a diet high in processed food and low in prebiotic fibres, bouts of stress and inactivity, exposure to antibiotics and environmental pollutants. The Bifidobacterial plays a key role in many areas of well-being and important for maintaining strong bones and joints, reducing temporary bouts of inflammation, maintaining strong memory and good mental health, promoting healthy digestion and nutrient absorption, helping body balance energy levels, protecting intestinal wall, and supporting the immune system. Further, having a balanced gut microbiome with Bifidobacterial species can control certain factors accelerating the shortening of telomeres [56].

\section{Caloric reduction with adequate nutrition (CRAN)}

The gut barrier and nutrient transport functions decline with aging, and dysbiosis weakens the intestinal barrier function. A moderate reduction in calorie intake, i.e., caloric restriction (CR) can potentially improve health and extend lifespan through certain evolutionarily conserved biological pathways. The evolutionarily conserved genes modulating aging in invertebrates and mammals, and linked to longevity in humans, include the insulin/IGF-1 like signaling (IIS) pathway, the target of rapamycin (TOR) and AMP-activated protein kinase (AMPK). 
The reduced nutrient availability decreases AKT activity resulting in enhanced FOXO activity. FOXO is abundantly expressed in the $\mathrm{C}$. elegans intestine, and its enhanced expression improves the lifespan.

Inversely, the activation of AKT (protein kinase B) by nutrient abundance leads to the inactivation of FOXO (a Forkhead box 0 transcription factor), which is a major factor to longevity, inducing expression of various pathways involved in cell metabolism, autophagy and stress-response. Another factor, FKH, a homolog of Forhhead box A (FOXA), is required for the IIS. Gut-specific FKH upregulation improves gut barrier function and expression of nutrient transporters, which help to increase lifespan, as in aged fruit flies.

Another nutrient signaling serine/threonine kinase pathway, mTOR (mechanistic or mammalian target of rapamycin), also responds to nutrients as well as certain other signals, such as growth hormones and mitogens. The mTOR as mTORC1 and mTORC2 (effects of mTORC1 are more extensive than mTORC2) subunits, both regulating various cellular processes related to cell growth, proliferation, and survival. FKH influences the mTOR pathway.

The health and lifespan extension by dietary intervention or inhibition of TOR activity by rapamycin involves altered gut microbiota, as shown in mice experiments. The gut microbiome and the host nutrient signaling pathways have been shown to be interconnected. The experiments using C. elegans and Escherichia coli-mutants have outlined the role of host IIS/TOR pathways for the lifespan extending effects.

\section{References}

1. Sender R, Fuchs S, Milo R (2016) Revised estimates for the number of human and bacteria cells in the body. PLoS Biol 14(8): e1002533.

2. O'Toole PW, Jeffery IB (2015) Gut microbiota and aging. Science 350(265): 1214-15

3. Flint HJ, Scott KP, Louis P, Duncan SH (2012) The role of the gut microbiota in nutrition and health. Nat Rev Gastroenterol Hepatol 9(10): 577-589.

4. Heintz C, Mair W (2014) You Are What You Host: Microbiome Modulation of the Aging Process. Cell 156(3): 408-411.

5. Kim S, Jazwinski SM (2018) The Gut Microbiota and Healthy Aging: A Mini-Review. Gerontology 64(6): 513-520.

6. Claesson MJ, Jeffery IB, Conde S, Susan E Power, Eibhlís M O'Connor, et al. (2012) Gut microbiota composition correlates with diet and health in the elderly. Nature 488: 178-184.

7. Clark RI, Salazar A, Yamada R, Fitz-Gibbon S, Morselli M, et al. 2015. Distinct shifts in microbiota composition during Drosophila aging impair intestinal function and drive mortality. Cell Rep 12(10): 16561667.

8. Kong F, Hua Y, Zeng B, Ning R, Li Y, et al. 2016. Gut microbiota signatures of longevity. Current Biology 26(18): R832-33.

9. Fontana L, Partridge L (2015) Promoting health and longevity through diet: From model organisms to humans. Cell 161(11): 106-118.

10. Jeffery IB, Lynch DB, O'Toole PW (2015) Composition and temporal stability of the gut microbiota in older persons. ISME J 10(1):170-182
11. Claesson MJ, Cusack S, O'Sullivan O, Greene-Diniz R, de Weerd H, et al. (2011) Composition, variability, and temporal stability of the intestinal microbiota of the elderly. Proc Natl Acad Sci USA 108 (suppl 1): 45864591.

12. Rampelli S, Candela M, Turroni S, Biagi E, Collino S, et al. (2013) Functional metagenomic profiling of intestinal microbiome in extreme ageing. Aging 5(12): 902-912.

13. Biagi E, Franceschi C, Simone RS, Severgnini M, Ostan R, et al. (2016) Gut Microbiota and Extreme Longevity. Curr Biol 26(11): 1480-1485.

14. Biagi E, Nylund L, Candela M, Ostan R, Bucci L, et al. (2010) Through ageing, and beyond: Gut microbiota and inflammatory status in seniors and centenarians. PLoS One 5(5): e10667.

15. Jackson MA, Jeffery IB, Beaumont M, Bell JT, Clark AG, et al. (2016) Signatures of early frailty in the gut microbiota. Genome Med. 8(1): 1-8.

16. Zhang R, Hou A (2013) Host-Microbe Interactions in Caenorhabditis elegans.

17. Gusarov I, Gautier L, Smolentseva O, Shamovsky I, Eremina S, et al. (2013) Bacterial nitric oxide extends the lifespan of C. elegans. Cell 152(4): 818-830.

18. Vaiserman AM, Koliada AK, Marotta F (2017) Gut microbiota: A player in aging and a target for anti-aging intervention. Ageing Res Rev 35: 36-45.

19. Buford TW (2017) (Dis)Trust your gut: the gut microbiome in age-related inflammation, health, and disease. Microbiome 5(80).

20. Smith P, Willemsen D, Popkes M, Metge F, Gandiwa E, et al. (2017) Regulation of life span by the gut microbiota in the short-lived African turquoise killifish. Elife 6: e27014.

21. Ren C, Webster P, Finkel SE, Tower J (2007) Increased internal and external bacterial load during Drosophila aging without life-span tradeoff. Cell Metab 6: 144-152.

22. Fransen F, van Beek AA, Borghuis T, Aidy SE, Hugenholtz F, et al. (2017) Aged gut microbiota contributes to systemical inflam-aging after transfer to germ-free mice. Front Immunol 8: 1385.

23. Sampson TR, Debelius JW, Thron T, Janssen S2, Shastri GG, et al. (2016) Gut microbiota regulate motor deficits and neuroinflammation in a model of Parkinson's disease. Cell 167(6): 1469-1480.

24. Blank M, Werenicz A, Velho LA, Pinto DF, Fedi AC, et al. (2015) Enhancement of memory consolidation by the histone deacetylase inhibitor sodium butyrate in aged rats. Neurosci Lett 594: 76-81.

25. Westfall S, Lomis N, Kahouli I, Dia SY, Singh SP, et al. (2017) Microbiome, probiotics and neurodegenerative diseases: deciphering the gut brain axis. Cell Mol Life Sci 74(20): 3769-3787.

26. Chandra R, Hiniker A, Kuo YM, Nussbaum RL, Liddle RA, et al. (2017) $\alpha$-Synuclein in gut endocrine cells and its implications for Parkinson's disease. JCI Insight 2(12): 92295.

27. Collins SM, Surette M, Bercik P (2012) The interplay between the intestinal microbiota and the brain. Nat Rev Microbiol, 10(11): 735-742.

28. Wen L, Duffy A (2017) Factors Influencing the Gut Microbiota, Inflammation, and Type 2 Diabetes. J Nutr 147(7): 1468S-1475S.

29. Tilg H, Moschen AR (2014) Microbiota and diabetes: an evolving relationship. Gut 63(9): 1513-1521.

30. Cattaneo A, Cattane N, Galluzzi S, Provasi S, Lopizzo N, et al. (2017) Association of brain amyloidosis with pro-inflammatory gut bacterial taxa and peripheral inflammation markers in cognitively impaired elderly. Neurobiol Aging 49: 60-68.

31. Cani PD, Osto M, Geurts L, Everard A (2012) Involvement of gut microbiota in the development of low-grade inflammation and type 2 diabetes associated with obesity. Gut Microbes 3(4): 279-288. 
32. Turnbaugh PJ, Ley RE, Mahowald MA, Magrini V, Mardis ER, et al. (2006) An obesity-associated gut microbiome with increased capacity for energy harvest. Nature 444: 1027-1031.

33. Burcelin R (2016) Gut microbiota and immune crosstalk in metabolic disease. Mol Metab 5: 771-781.

34. Hersoug LG, Moller P, Loft S (2016) Gut microbiota-derived lipopolysaccharide uptake and trafficking to adipose tissue: implications for inflammation and obesity. Obes Rev 17(4): 297-312.

35. Meijer K, de Vos P, Priebe MG (2010) Butyrate and other short-chain fatty acids as modulators of immunity: what relevance for health? Curr Opin Clin Nutr Metab Care 13: 715-721.

36. Le Chatelier E, Nielsen T, Qin J, Edi Prifti, Falk Hildebrand, et al. (2013) Richness of human gut microbiome correlates with metabolic markers. Nature 500: 541-546.

37. Blaser MJ, Falkow S (2009) What are the consequences of the disappearing human microbiota? Nat Rev Microbiol 7(12): 887-894.

38. Man AL, Bertelli E, Rentini S, Briars G, Marini M, et al. (2015) Age-associated modifications of intestinal permeability and innate immunity in human small intestine. Clin Sci 129(7): 515-527.

39. Cusack S, O’Toole PW (2012) Challenges and Implications for Biomedical Research and Intervention Studies in Older Populations: Insights from the ELDERMET Study. Gerontology 59(2): 114-121.

40. Thevaranjan N, Puchta A, Schulz C, Naidoo A, Szamosi JC, et al. (2017) Age-Associated Microbial Dysbiosis Promotes Intestinal Permeability, Systemic Inflammation, and Macrophage Dysfunction. Cell Host Microbe 21(4): 455-466.

41. Leung K, Thuret S (2015) Gut microbiota: a modulator of brain plasticity and cognitive function in ageing. Healthcare 3(4): 898-916.

42. Lloyd-Price J, Abu-Ali G, Huttenhower C (2016) The healthy human microbiome. Genome Med 8(1): 51.

43. De Filippis F, Pellegrini N, Vannini L, Jeffery IB, La Storia A, et al. (2016) High-level adherence to a Mediterranean diet beneficially impacts the gut microbiota and associated metabolome. metabolome Gut 65(11): 1812-1821.

44. Kenyon CJ (2010) The genetics of ageing. Nature 464: 504-512.
45. Clarke SF, Murphy EF, O’Sullivan O, Lucey AJ, Humphreys M, et al. (2014) Exercise and associated dietary extremes impact on gut microbial diversity. Gut 63(12): 1913-1920.

46. Graf D, Di Cagno R, Fak F, Nyman M, Saarela M, et al. (2015) Contribution of diet to the composition of the human gut microbiota. Microb Ecol Health Dis 26: 261-264.

47. Olivares M, Paz Diaz-Ropero M, Gomez N, Sierra S, Lara-Villoslada F, et al. (2006) Dietary deprivation of fermented foods causes a fall in innate immune response. Lactic acid bacteria can counteract the immunological effect of this deprivation. J Dairy Res 73(4): 492-498.

48. Mackowiak PA (2013) Recycling metchnikoff: probiotics, the intestinal microbiome and the quest for long life. Front Public Health 1: 52.

49. van Beek AA, Sovran B, Hugenholtz F, Meijer B, Hoogerland JA, et al. (2016) Supplementation with Lactobacillus plantarum WCFS1 Prevents Decline of Mucus Barrier in Colon of Accelerated Aging Ercc1-/ Delta7 Mice. Front Immunol 7: 408.

50. Kumar M, Yadav AK, Verma V, Singh B, Mal G, et al. (2016) Bioengineered probiotics as a new hope for health and diseases: an overview of potential and prospects. Future Microbiol 11(4): 585-600.

51. Lee H, Ko G (2014) Effect of metformin on metabolic improvement and gut microbiota. Appl Environ Microbiol 80: 5935-5943.

52. Forslund K, Hildebrand F, Nielsen T, Falony G, Le Chatelier E, et al. (2015) Disentangling type 2 diabetes and metformin treatment signatures in the human gut microbiota. Nature 528(7581): 262-266.

53. Shammas MA (2011) Telomeres, lifestyle, cancer, and aging. Curr Opin Clin Nutr Metab Care 14(1): 28-34.

54. Bian G, Gloor GB, Gong A, Jia C, Zhang W, et al. (2017) The Gut Microbiota of Healthy Aged Chinese Is Similar to That of the Healthy Young. mSphere 2(5): e00327-17.

55. Salazar N, Valdés-Varela L, González S, Gueimonde M, de los Reyes-Gavilána CG (2017) Nutrition and the Gut Microbiome in the Elderly. Gut Microbes 8(2): 82-97.

56. Matsumoto M, Kurihara S (2011) Probiotics-induced Increase of Large Intestinal Luminal Polyamine Concentration May Promote Longevity. Medical Hypotheses 77(4): 469-472.

\section{Your next submission with Juniper Publishers} will reach you the below assets

- Quality Editorial service

- Swift Peer Review

- Reprints availability

- E-prints Service

- Manuscript Podcast for convenient understanding

- Global attainment for your research

- Manuscript accessibility in different formats

(Pdf, E-pub, Full Text, Audio)

- Unceasing customer service

Track the below URL for one-step submission https://juniperpublishers.com/online-submission.php 